\title{
"Softness" as the structural origin of plasticity in disordered solids: a quantitative insight from machine learning
}

\author{
Xiaodi Liu ${ }^{1}$, Fucheng $\mathrm{Li}^{1}$ and Yong Yang ${ }^{1,2^{*}}$
}

Disordered solids, including various types of glasses, such as metallic glasses (MGs), ceramic glasses and polymeric glasses, possess an amorphous or aperiodic arrangement of their building blocks, such as atoms, ions and molecules. At first sight, this is similar to the structure of their corresponding liquids, lacking any long-range translational symmetry as in crystalline solids. As a result, disordered solids usually exhibit a unique combination of properties, and are an indispensable class of engineering materials. For instance, MGs exhibit superb strength, high elastic limit, and outstanding corrosion- and wearresistance, which is ideal for making sporting and medical apparatus. However, disordered solids, particularly structural glasses, could be very brittle under an ambient condition. Catastrophic failures can take place in them without noticeable plasticity $[1,2]$. In practice, this severely hinders the applications of disordered solids as a reliable structural material; therefore, the origin of plasticity in disordered solids, if any, has been remaining in decades as one of the most active topics in materials science and engineering.

Unlike crystalline solids, disordered solids possess no well-defined structural defects, which can easily "migrate" to initiate a plastic flow, as an analogue to dislocations in crystalline solids. To understand the origin of plasticity in disordered solids, tremendous amounts of efforts have been made, with various models and theories being developed over the past decades, such as free volume model [3], shear transformation zone (STZ) model [4,5] and flow unit model [6]. Today, the consensus is that plasticity in disordered solids may be rooted in some loosely packed regions, which behave like a flow "defect". However, many details about these flow "defects", such as their size, still remains to be fully resolved.
In a recent paper published in Science by Cubuk et al. [7], the authors defined a microstructural quantity, termed "softness", based on a supervised machine learning model [8]. To determine the "softness" of particle $i$, a set of structural functions are defined, as listed below:

$$
\begin{gathered}
G(i ; \mu)=\sum_{k} \mathrm{e}^{-\left(r_{i k}-\mu\right)^{2} / L^{2}}, \\
\Psi(i ; \xi, \lambda, \zeta)=\sum_{j, k} \mathrm{e}^{-\left(r_{i k}^{2}+r_{j k}^{2}+r_{i j}^{2}\right) / \xi^{2}}\left(1+\lambda \cos \theta_{k i j}\right)^{\zeta},
\end{gathered}
$$

where $r_{i k}$ is the distance between particle $i$ and $k$; $\theta_{k i j}$ is the angle spanned between the two vectors $r_{i j}$ and $r_{i k}$. Here, $\mu$, $\xi, \lambda, L$ and $\zeta$ are all parameters related to particles, as defined in Ref. [7]. For data training, one has to determine particles that are prone to rearrange their positions with their neighbors. In a mechanically loaded system, this is done by tracking the particles with their non-affine displacement greater than a pre-defined threshold $D_{\min , 0}^{2}$ at a given time [5]. Or, in a quiescent system, one defines the quantity $p_{\text {hop }}$, which reads:

$$
\begin{aligned}
& p_{\text {hop }}(i, t) \\
& \quad=\sqrt{\left\langle\left(\vec{r}_{i}(t)-\left\langle\vec{r}_{i}(t)\right\rangle_{B}\right)^{2}\right\rangle_{A}\left\langle\left(\vec{r}_{i}(t)-\left\langle\vec{r}_{i}(t)\right\rangle_{A}\right)^{2}\right\rangle_{B}},
\end{aligned}
$$

where $A=[t-\delta t / 2, t], B=[t, t+\delta t / 2],\langle\cdot\rangle_{A}$ and $\langle\cdot\rangle_{B}$ are the expectation values over the $A$ and $B$ intervals. In principle, the particles with $p_{\text {hop }}$ above a threshold value of $p_{\text {hop }, 0}$ are considered to be rearranging. Based on the obtained training data from either a mechanically loaded or a quiescent system, a hyperplane can be constructed in the structure function space defined through Equations $(1,2)$, which best separates particles that tend to rearrange themselves from those that tend not to.

Consequently, the "softness", $S_{k}$, of an arbitrary particle

\footnotetext{
${ }^{1}$ Department of Mechanical Engineering, City University of Hong Kong, Kowloon, Hong Kong, China

${ }^{2}$ Centre for Advanced Structural Materials, City University of Hong Kong, Kowloon, Hong Kong, China

*Corresponding author (email: yonyang@cityu.edu.hk)
} 
a

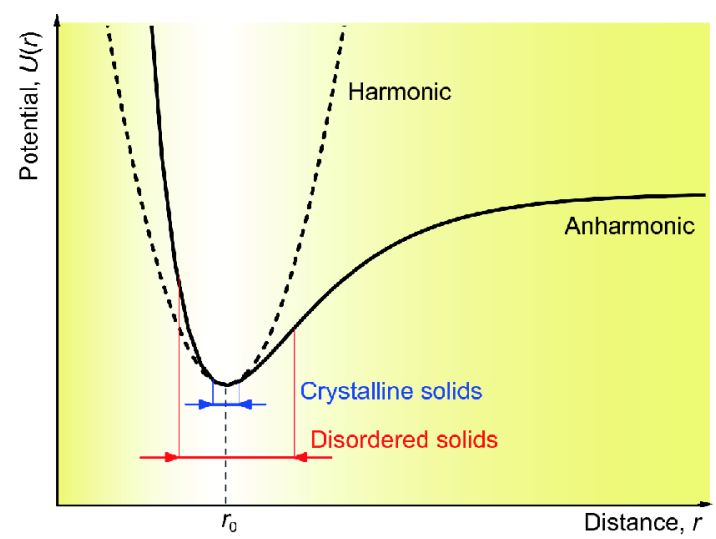

b

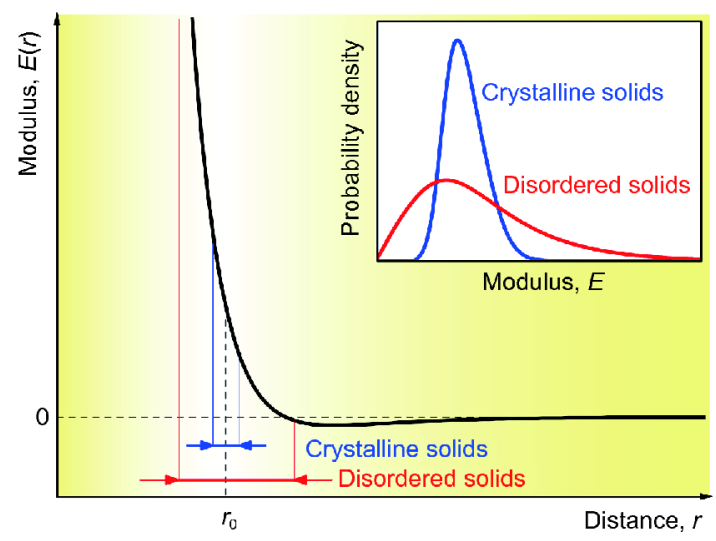

Figure 1 Variation of interatomic potential and modulus with interatomic distance. (a) $U(r)$ vs. $r . r_{0}$ is the equilibrium position with the minimum value of $U(r)$. The dash line stands for an approximation by a harmonic oscillator potential at $r=r_{0}$. (b) $E(r) v s$. $r$. The inset shows the distribution of moduli.

$k$ can be computed based on the distance of the particle's position in the structure-function space to the hyperplane. If a particle lies on the same side of the hyperplane as the rearranging particles in the training set, it is considered to possess "softness". In principle, the particles with higher "softness" show a better spatial correlation with the regions undergoing large rearrangements. More importantly, Cubuk and co-workers [7] discovered that the normalized spatial correlation function $\langle\delta S(0) \delta(r)\rangle \equiv\left(\langle S(0) S(r)\rangle-\langle S\rangle^{2}\right) /\left(\left\langle S^{2}\right\rangle-\langle S\rangle^{2}\right)$ shows an exponential decay as expressed by $\langle\delta S(0) \delta(r)\rangle=\exp \left(-r / \xi_{\mathrm{s}}\right)$, and the correlation length $\xi_{\mathrm{s}}$ has the following scaling relation to the particle size $(d)$ for different kinds of disordered solids:

$$
\xi_{\mathrm{s}} / d=1.1 \pm 0.2
$$

Furthermore, the authors found that "softness" evolves with strain in various disordered solids. Yielding is trig- gered when the change in "softness" reaches a common value of $0.07 \pm 0.02$, which corresponds to a common yield strain $\varepsilon_{y}=2.9 \pm 0.3 \%$. These findings are of great importance, which indicates that plasticity in disordered solids, regardless of their chemical makeup, could have a common structural origin.

Prior to the "softness" proposed by Cubuk et al. [7], there are already a number of theories or models developed in the literature to explain the origin of plasticity in disordered solids. These include the free volume model originally proposed by Cohen and Turnbull [9] and developed by Spaepen et al. [3], which defined free volume as the excess space among atoms and assumed that plastic flow should result from a number of individual atomic jumps in and out of free volume. Argon et al. $[4,10]$ later developed the STZ model, ascribing plasticity in disordered solids to the collective atomic slips involving tens of atoms. Falk and Langer [5,11] conjectured that STZ should be some pre-existing flow "defects", which comprise a group of atoms susceptible to structural rearrangements upon external agitations. A similar idea of pre-existing flow defects can be also found in the random first order transition theory [12], the flow unit model of Wang et al. [6] and others [13-16]. In general, according to these theories and conjectures, disordered solids or structural glass may be deemed as a composite made up of a "solid-like" matrix and "liquid-like" inclusions or flow units. Following this line of reasoning, one may picture that plastic flow in disordered solids should be caused by the percolation of the local flow defects. This is a scenario that can be envisioned based on the view that disordered solids are intrinsically heterogeneous [17-19], containing soft spots or local regions of significant "physical softness".

At the fundamental level, physical softness in disordered solids may be understood from the perspective of an inter-particle potential. For MGs, the simplest form of disordered solids (also called "atomic glass"), this is related to the interatomic potential $U(r)$ as illustrated in Fig. 1a, where $r$ denotes the inter-atomic distance. For an ideal crystalline solid, its $U(r)$ reaches the minimum potential energy and, theoretically, the inter-atomic distance should be uniform and equal to the equilibrium bond length $r_{0}$. Elastic deformation then causes the interatomic distance to deviate from $r_{0}$, which gives $\varepsilon_{\mathrm{E}}=\left(r-r_{0}\right) /$ $r_{0}$, where $\varepsilon_{\mathrm{E}}$ is the elastic strain and $r$ is the instantaneous bond length. In this case, the potential energy $U(r)$ of the stressed crystalline solid may be approximated as that for a harmonic oscillator or $U(r) \sim\left(r-r_{0}\right)^{2}$. In general, one can readily derive the elastic modulus $E$ of the solid from its 

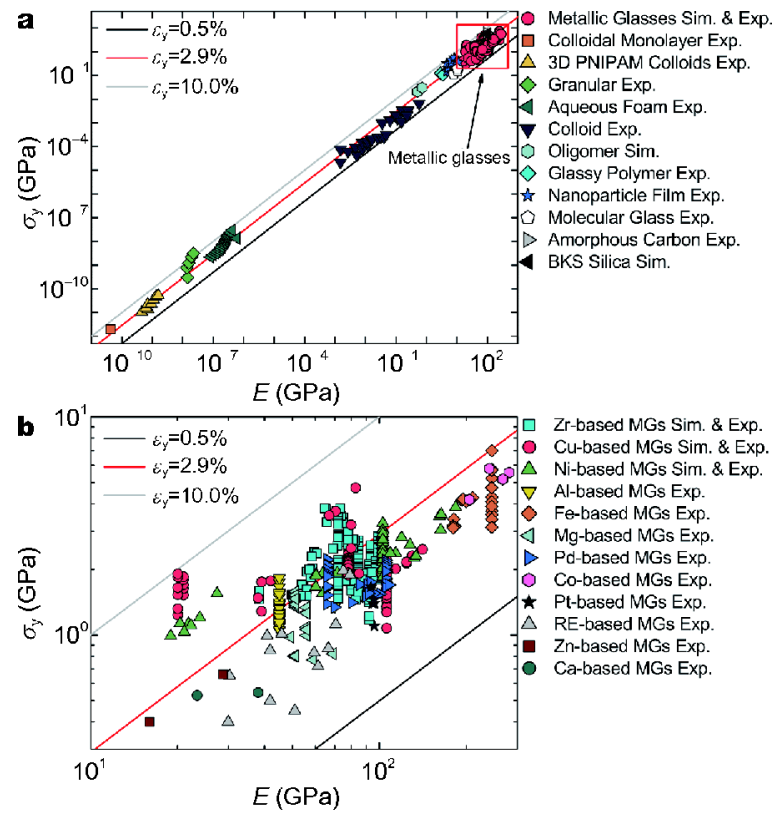

Figure 2 Relationship between yield strength $\left(\sigma_{y}\right)$ and Young's modulus (E). (a) A general rule for a variety of disordered solids. (b) Enlarged view of the box in (a), to display the details of metallic glasses $[8,28,30,33-34,47-61]$.

potential energy $U(r)$ :

$$
E \sim \frac{\partial^{2} U(r)}{r \partial r^{2}}
$$

In sharp contrast, a disordered solid is not in a thermodynamically equilibrium state. Therefore, the distribution of the interatomic distance in it could be much broader than that in its crystalline counterpart, as confirmed by the neutron diffraction experimental results [20]. In this case, local regions with atomic bonds much longer or shorter than $r_{0}$ possess a higher potential energy or may appear softer in their local elastic moduli. As one can infer from Equation (5), the local elastic modulus could be negative in the regions where $\partial^{2} U(r) / \partial r^{2}<0$. This means that some local regions in a disordered solid may not be able to take stress as an ordinary solid and therefore behave like a liquid. This defines the "anharmonicity" with respect to the behavior of atoms in a disordered solid. When anharmonicity is considered, the harmonic approximation of $U(r)$ is not valid any more. Instead, structural heterogeneity or "softness" must be taken into account. Fig. 1b evidently illustrates that $E$ varies with $r$ and the distribution of moduli for both crystalline and disordered solids. Please note that the mechanical softness derived from Equation (5) differs fundamentally from the softness of Cubuk et al. [7]; however, these two should have a good correlation given that both are also correlated with the non-affine deformation in disordered solids under mechanical stress.

Generally, crystalline solids, such as crystalline metals and alloys, start to yield at a stress $<0.005 E$, which is far below the theoretical elastic limit $(\sim 0.1 E)$ evaluated by Frenkel [21]. By contrast, disordered solids exhibit ultrahigh elastic limit. As shown in Fig. 2a, there seems to be a general rule between the yield strength $\left(\sigma_{y}\right)$ and Young's modulus $(E)$ of a variety of disordered solids, covering $>10$ orders of magnitude. In spite of the scatter in data, a linear fitting is able to provide a common yield strain $\varepsilon_{\mathrm{y}} \sim 2.9 \%$ [7], which is consistent with the average shear strain limit $\gamma_{\mathrm{y}} \sim 2.7 \%$ for a multitude of MGs [22,23]. Furthermore, Cubuk et al. [7] discovered that the common yield strain $\varepsilon_{y} \sim 2.9 \%$ corresponds to a common change in "softness" $\Delta S(\varepsilon) \sim 0.07$. This finding agrees with the view that yielding in disordered solids is a result of percolation of some follow defects. In theory, Egami et al. $[24,25]$ connected the microscopic mechanism of plastic flow with that of glass transition, and proposed a similar mechanism of yielding that yielding is caused by the fraction of liquid-like sites, which can be defined by topological instability, exceeding the percolation limit of the system, which is thermodynamically analogous to a stress induced glass-to-liquid transition $[25,26]$. From the perspective of potential energy landscape (PEL), each individual metabasin represents a local configuration, which can be characterized by local elastic modulus [22]. In this scenario, the elastic-plastic transition is given by the percolation of "soft spots" within the distribution of elastic moduli and therefore local "soft" configurations [27]. Here, we show an enlarged view in Fig. $2 b$ to look into more details of the yield strengths of MGs. Evidently, the reported data do not perfectly collapse onto the same trend line but are scattered between the $1 \%$ and $10 \%$ bounds. Cubuk et al. [7] also noticed this and attributed it to a possible size effect, emphasizing that $\varepsilon_{\mathrm{y}} \sim 2.9 \%$ holds only for a macroscopic behavior. Besides the size effect [28-34], many other factors may also play a role for the variation of $\varepsilon_{y}$, such as the processing history [35-40] and chemical heterogeneity $[41,42]$.

The distinct origin of plasticity in disordered solids compared to their crystalline counterparts also manifests in the magnitude of their yield strengths. For crystalline solids, plasticity stems from the movement of lattice defects, such as dislocations, as illustrated in Fig. 3a-c. During the dislocation migration process, only a small fraction of bonds are broken at any given time, and thus energy barrier against this dislocation migration could be 

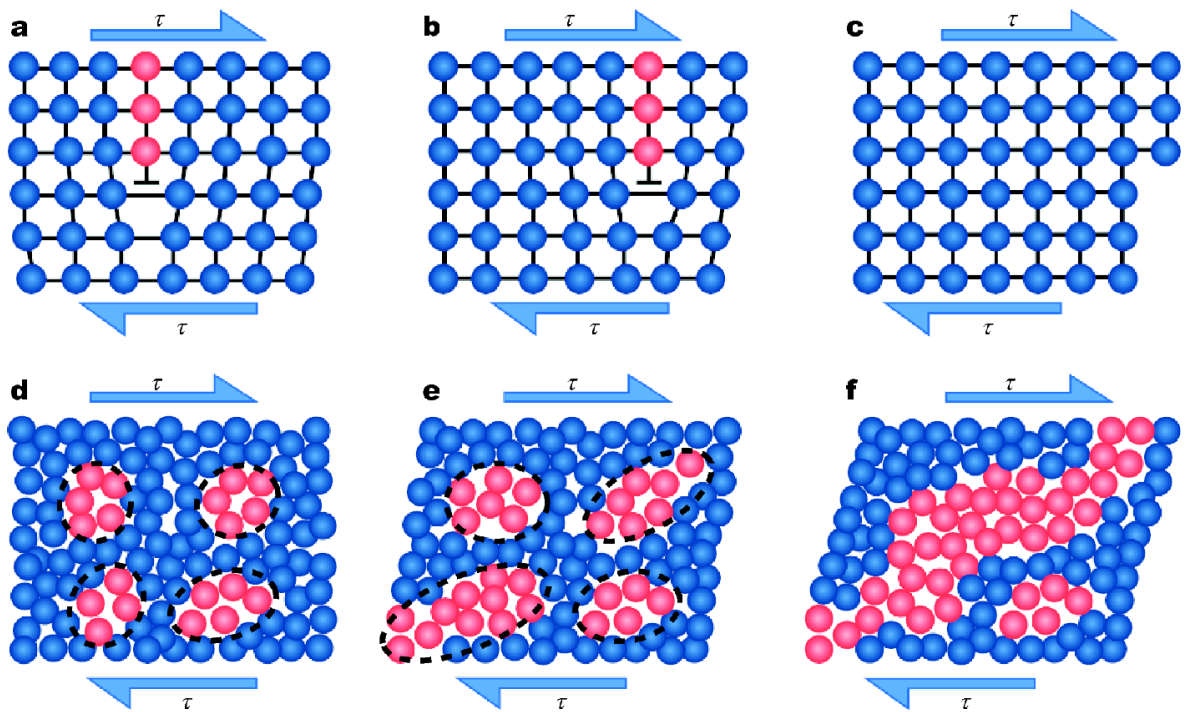

Figure 3 Illustrations of the mechanisms of the plastic deformation in $(\mathrm{a}-\mathrm{c})$ crystalline solids and $(\mathrm{d}-\mathrm{f})$ disordered solids. For crystalline solids, red spheres represent the atoms in the extra-half plane, and " $\perp$ " stands for the edge dislocation line. The migration of dislocation generates the plastic strain. For disordered solids, the red spheres stand for the "defect" regions, which grow and coalesce under shear stress, leading to local atomic rearrangements. The percolation through the "matrix" composed by the blue spheres causes overall softening and plastic flow.

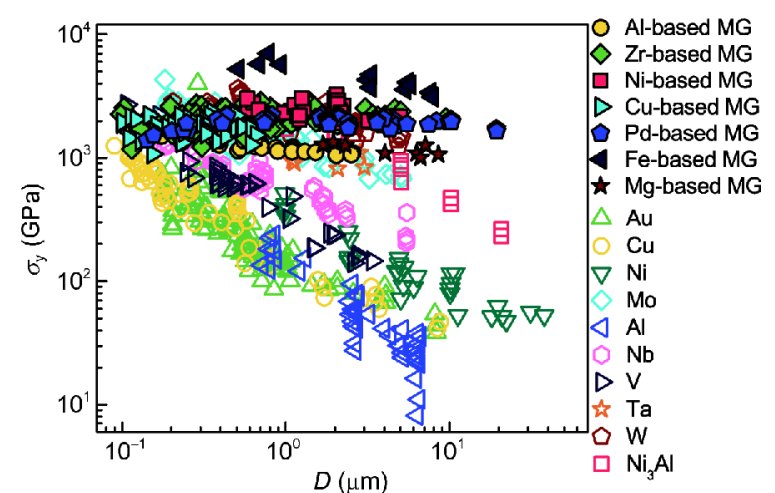

Figure 4 The comparison of strength size effect between crystal and non-crystal materials $[30,33,34,43,47-50,55,62-69]$.

small, resulting in a low yield strength. On the contrary, plastic flows in disordered solids initiate from "defects" with large "softness" and low moduli, which initially are isolated and dispersed in the "stiff" amorphous matrix (Fig. 3d). Under the applied shear stress, the "defect" regions grow and coalesce, leading to local atomic rearrangements (Fig. 3e). Eventually, these "defects" percolate through the "matrix", causing overall softening and plastic flows (Fig. 3f). At low temperature or high strain rate, this mechanical softening usually occurs at a relatively high yield strength, which is accompanied by plastic flow localization or shear banding $[1,3]$.

Because of the different plasticity mechanism, crystal- line metals usually exhibit a significant strength enhancement when their sample size decreases from bulk to a sub-micron scale while disordered metals do not, as seen clearly in Fig. 4a. Such a phenomenon of "smallerbeing-stronger" for crystalline metals is often attributed to the interplay of dislocation motion with free surfaces, such as "dislocation starvation" proposed in Ref. [43]. By comparison, the size effect in MGs is not as significant as in crystals and even appears stochastic in micro-compression $[44,45]$. At the present time, it is still an ongoing research to understand the strength size effect in disordered solids $[28,30,46-50]$.

To sum up, the work of Cubuk [7] demonstrates that the "softness" defined through machine learning is a good metric to probe the structural origin of plasticity initiation in an amorphous solid. Nevertheless, the physical meaning of the machine learning deduced "softness" is still not clear. In our view, it could be an interesting topic in near future to study the correlation, if there is any, between the machine learning deduced "softness" and the physical "softness" of particles in an amorphous solid, through which one could finally decode the physical origin of plasticity in disordered solids.

Received 23 April 2018; accepted 21 June 2018;

published online 3 July 2018

1 Schuh C, Hufnagel T, Ramamurty U. Mechanical behavior of amorphous alloys. Acta Mater, 2007, 55: 4067-4109 
2 Telford M. The case for bulk metallic glass. Mater Today, 2004, 7: 36-43

3 Spaepen F. A microscopic mechanism for steady state inhomogeneous flow in metallic glasses. Acta Metall, 1977, 25: 407415

4 Argon AS. Plastic deformation in metallic glasses. Acta Metall, 1979, 27: 47-58

5 Falk ML, Langer JS. Dynamics of viscoplastic deformation in amorphous solids. Phys Rev E, 1998, 57: 7192-7205

6 Lu Z, Jiao W, Wang WH, et al. Flow unit perspective on room temperature homogeneous plastic deformation in metallic glasses. Phys Rev Lett, 2014, 113: 045501

7 Cubuk ED, Ivancic RJS, Schoenholz SS, et al. Structure-property relationships from universal signatures of plasticity in disordered solids. Science, 2017, 358: 1033-1037

8 Suykens JAK, Vandewalle J. Least squares support vector machine classifiers. Neural Proc Lett, 1999, 9: 293-300

9 Cohen MH, Turnbull D. Molecular transport in liquids and glasses. J Chem Phys, 1959, 31: 1164-1169

10 Argon AS, Kuo HY. Plastic flow in a disordered bubble raft (an analog of a metallic glass). Mater Sci Eng, 1979, 39: 101-109

11 Langer J. Shear-transformation-zone theory of deformation in metallic glasses. Scripta Mater, 2006, 54: 375-379

12 Xia X, Wolynes PG. Fragilities of liquids predicted from the random first order transition theory of glasses. Proc Natl Acad Sci USA, 2000, 97: 2990-2994

13 Ye JC, Lu J, Liu CT, et al. Atomistic free-volume zones and inelastic deformation of metallic glasses. Nat Mater, 2010, 9: 619-623

14 Liu ZY, Yang Y. A mean-field model for anelastic deformation in metallic-glasses. Intermetallics, 2012, 26: 86-90

15 Ding J, Patinet S, Falk ML, et al. Soft spots and their structural signature in a metallic glass. Proc Natl Acad Sci USA, 2014, 111: 14052-14056

16 Wang Z, Sun BA, Bai HY, et al. Evolution of hidden localized flow during glass-to-liquid transition in metallic glass. Nat Commun, 2014, 5: 5823

17 Liu YH, Wang D, Nakajima K, et al. Characterization of nanoscale mechanical heterogeneity in a metallic glass by dynamic force microscopy. Phys Rev Lett, 2011, 106: 125504

18 Hu YC, Guan PF, Li MZ, et al. Unveiling atomic-scale features of inherent heterogeneity in metallic glass by molecular dynamics simulations. Phys Rev B, 2016, 93: 214202

19 Huo LS, Zeng JF, Wang WH, et al. The dependence of shear modulus on dynamic relaxation and evolution of local structural heterogeneity in a metallic glass. Acta Mater, 2013, 61: 4329-4338

20 Fan C, Liaw PK, Haas V, et al. Structures and mechanical behaviors of $\mathrm{Zr}_{55} \mathrm{Cu}_{35} \mathrm{Al}_{10}$ bulk amorphous alloys at ambient and cryogenic temperatures. Phys Rev B, 2006, 74: 014205

21 Frenkel J. Zur Theorie der Elastizitätsgrenze und der Festigkeit kristallinischer Körper. Zeitschrift für Physik, 1926, 37: 572-609

22 Johnson WL, Samwer K. A universal criterion for plastic yielding of metallic glasses with a $\left(T / T_{\mathrm{g}}\right)^{2 / 3}$ temperature dependence. Phys Rev Lett, 2005, 95: 195501

23 Qu RT, Liu ZQ, Wang RF, et al. Yield strength and yield strain of metallic glasses and their correlations with glass transition temperature. J Alloys Compd, 2015, 637: 44-54

24 Egami T, Poon SJ, Zhang Z, et al. Glass transition in metallic glasses: A microscopic model of topological fluctuations in the bonding network. Phys Rev B, 2007, 76: 024203
Guan P, Chen M, Egami T. Stress-temperature scaling for steadystate flow in metallic glasses. Phys Rev Lett, 2010, 104: 205701

27 Wagner H, Bedorf D, Küchemann S, et al. Local elastic properties of a metallic glass. Nat Mater, 2011, 10: 439-442

28 Li FC, Wang S, He QF, et al. The stochastic transition from size dependent to size independent yield strength in metallic glasses. J Mech Phys Solids, 2017, 109: 200-216

29 Wang S, Ye YF, Wang Q, et al. The breakdown of strength size scaling in spherical nanoindentation and microcompression of metallic glasses. Scripta Mater, 2017, 130: 283-287

30 Wang CC, Ding J, Cheng YQ, et al. Sample size matters for $\mathrm{Al}_{88} \mathrm{Fe}_{7} \mathrm{Gd}_{5}$ metallic glass: Smaller is stronger. Acta Mater, 2012, 60: 5370-5379

31 Schuster BE, Wei Q, Ervin MH, et al. Bulk and microscale compressive properties of a Pd-based metallic glass. Scripta Mater, 2007, 57: 517-520

32 Liontas R, Jafary-Zadeh M, Zeng Q, et al. Substantial tensile ductility in sputtered $\mathrm{Zr}-\mathrm{Ni}$-Al nano-sized metallic glass. Acta Mater, 2016, 118: 270-285

33 Jang D, Gross CT, Greer JR. Effects of size on the strength and deformation mechanism in Zr-based metallic glasses. Int J Plasticity, 2011, 27: 858-867

34 Bharathula A, Flores KM. Variability in the yield strength of a metallic glass at micron and submicron length scales. Acta Mater, 2011, 59: 7199-7205

35 Bian XL, Wang G, Chen HC, et al. Manipulation of free volumes in a metallic glass through Xe-ion irradiation. Acta Mater, 2016, 106 : 66-77

36 Baumer RE, Demkowicz MJ. Radiation response of amorphous metal alloys: Subcascades, thermal spikes and super-quenched zones. Acta Mater, 2015, 83: 419-430

37 Magagnosc DJ, Ehrbar R, Kumar G, et al. Tunable tensile ductility in metallic glasses. Sci Rep, 2013, 3: 1096

38 Magagnosc DJ, Kumar G, Schroers J, et al. Effect of ion irradiation on tensile ductility, strength and fictive temperature in metallic glass nanowires. Acta Mater, 2014, 74: 165-182

39 Yu HB, Luo Y, Samwer K. Ultrastable metallic glass. Adv Mater, 2013, 25: 5904-5908

40 Magagnosc DJ, Feng G, Yu L, et al. Isochemical control over structural state and mechanical properties in Pd-based metallic glass by sputter deposition at elevated temperatures. APL Mater, 2016, 4: 086104

41 Chen LY, Fu ZD, Zhang GQ, et al. New class of plastic bulk metallic glass. Phys Rev Lett, 2008, 100: 075501

42 Kim KB, Das J, Venkataraman S, et al. Work hardening ability of ductile $\mathrm{Ti}_{45} \mathrm{Cu}_{40} \mathrm{Ni}_{7.5} \mathrm{Zr}_{5} \mathrm{Sn}_{2.5}$ and $\mathrm{Cu}_{47.5} \mathrm{Zr}_{47.5} \mathrm{Al}_{5}$ bulk metallic glasses. Appl Phys Lett, 2006, 89: 071908

43 Greer JR, Oliver WC, Nix WD. Size dependence of mechanical properties of gold at the micron scale in the absence of strain gradients. Acta Mater, 2005, 53: 1821-1830

44 Gao Y, Bei H. Strength statistics of single crystals and metallic glasses under small stressed volumes. Prog Mater Sci, 2016, 82: $118-150$

45 Greer JR, De Hosson JTM. Plasticity in small-sized metallic systems: Intrinsic versus extrinsic size effect. Prog Mater Sci, 2011, 56: 654-724

46 Jang D, Greer JR. Transition from a strong-yet-brittle to a stronger-and-ductile state by size reduction of metallic glasses. Nat Mater, 2010, 9: 215-219

47 Bharathula A, Lee SW, Wright WJ, et al. Compression testing of 
metallic glass at small length scales: Effects on deformation mode and stability. Acta Mater, 2010, 58: 5789-5796

48 Chen CQ, Pei YT, De Hosson JTM. Effects of size on the mechanical response of metallic glasses investigated through in situ TEM bending and compression experiments. Acta Mater, 2010, 58: 189-200

49 Kuzmin OV, Pei YT, Chen CQ, et al. Intrinsic and extrinsic size effects in the deformation of metallic glass nanopillars. Acta Mater, 2012, 60: 889-898

50 Ye JC, Lu J, Yang Y, et al. Extraction of bulk metallic-glass yield strengths using tapered micropillars in micro-compression experiments. Intermetallics, 2010, 18: 385-393

51 Wang WH. The elastic properties, elastic models and elastic perspectives of metallic glasses. Prog Mater Sci, 2012, 57: 487-656

52 Dubach A, Raghavan R, Loffler J, et al. Micropillar compression studies on a bulk metallic glass in different structural states. Scripta Mater, 2009, 60: 567-570

53 Lai YH, Lee CJ, Cheng YT, et al. Bulk and microscale compressive behavior of a Zr-based metallic glass. Scripta Mater, 2008, 58: 890893

54 Lee CJ, Huang JC, Nieh TG. Sample size effect and microcompression of $\mathrm{Mg}_{65} \mathrm{Cu}_{25} \mathrm{Gd}_{10}$ metallic glass. Appl Phys Lett, 2007, 91: 161913

55 Schuster BE, Wei Q, Hufnagel TC, et al. Size-independent strength and deformation mode in compression of a Pd-based metallic glass. Acta Mater, 2008, 56: 5091-5100

56 Volkert CA, Donohue A, Spaepen F. Effect of sample size on deformation in amorphous metals. J Appl Phys, 2008, 103: 083539

57 Zhou X, Zhou H, Li X, et al. Size effects on tensile and compressive strengths in metallic glass nanowires. J Mech Phys Solids, 2015, 84: 130-144

58 Şopu D, Foroughi A, Stoica M, et al. Brittle-to-ductile transition in metallic glass nanowires. Nano Lett, 2016, 16: 4467-4471

59 Delogu F. Molecular dynamics study of size effects in the compression of metallic glass nanowires. Phys Rev B, 2009, 79: 184109

60 Zhao P, Li J, Wang Y. Extended defects, ideal strength and actual strengths of finite-sized metallic glasses. Acta Mater, 2014, 73: 149-
166

61 Chen DZ, Jang D, Guan KM, et al. Nanometallic glasses: size reduction brings ductility, surface state drives its extent. Nano Lett, 2013, 13: 4462-4468

62 Uchic MD, Dimiduk DM, Florando JN, et al. Sample dimensions influence strength and crystal plasticity. Science, 2004, 305: 986989

63 Dimiduk DM, Uchic MD, Parthasarathy TA. Size-affected singleslip behavior of pure nickel microcrystals. Acta Mater, 2005, 53: 4065-4077

64 Volkert CA, Lilleodden ET. Size effects in the deformation of submicron Au columns. Philos Mag, 2006, 86: 5567-5579

65 Brinckmann S, Kim JY, Greer JR. Fundamental differences in mechanical behavior between two types of crystals at the nanoscale. Phys Rev Lett, 2008, 100: 155502

$66 \mathrm{Ng}$ KS, Ngan AHW. Stochastic theory for jerky deformation in small crystal volumes with pre-existing dislocations. Philos Mag, 2008, 88: 677-688

67 Jennings AT, Burek MJ, Greer JR. Microstructure versus size: mechanical properties of electroplated single crystalline $\mathrm{Cu}$ nanopillars. Phys Rev Lett, 2010, 104: 135503

68 Han SM, Bozorg-Grayeli T, Groves JR, et al. Size effects on strength and plasticity of vanadium nanopillars. Scripta Mater, 2010, 63: 1153-1156

69 Kiener D, Minor AM. Source truncation and exhaustion: insights from quantitative in situ TEM tensile testing. Nano Lett, 2011, 11: 3816-3820

Acknowledgements Yang Y acknowledges the research funding from Research Grant Council (RGC) of Hong Kong with the grant number CityU 11207215 and CityU11209317.

Author contributions Liu X, Li F and Yang Y wrote the paper. All authors contributed to the general discussion.

Conflict of interest The authors declare no conflict of interest. 

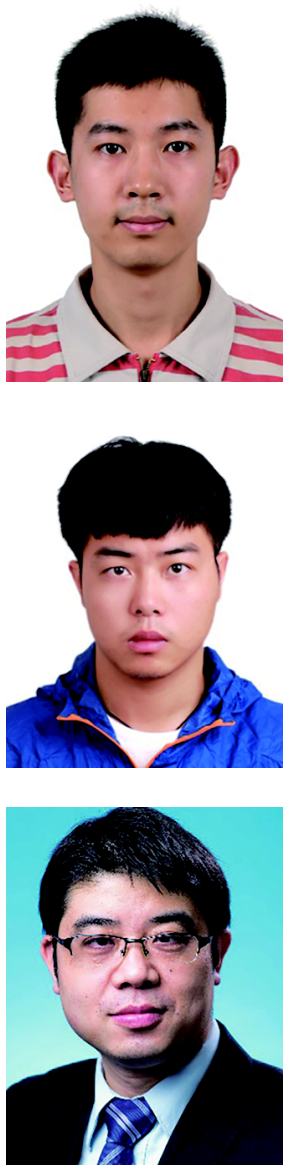

Xiaodi Liu obtained his bachelor and master degree from Shandong University, Jinan, China, in 2012 and 2015 respectively. He is currently a PhD student under the supervision of Prof. Yong Yang at the Department of Mechanical Engineering, City University of Hong Kong, Hong Kong, China. His research focuses on the creep and relaxation behavior of metallic glasses.

Fucheng Li is currently a PhD candidate at the Department of Mechanical Engineering, City University of Hong Kong, Hong Kong, China. He received his bachelor degree and master degree from Central South University, Changsha, China in 2013 and 2016, respectively. His $\mathrm{PhD}$ research focuses on the mechanical behavior of both bulk nano-grained metallic glass and metallic-glass based nanostructures.

Yong Yang obtained his bachelor degree in 2001 from Peking University, Beijing, China, and PhD in 2007 from Princeton University, NJ, USA. He is currently a Professor at the Department of Mechanical Engineering, City University of Hong Kong, Hong Kong, China. His research focuses on mechanical behavior of structural materials and the high throughput design of alloys, such as metallic glasses and high entropy alloys.

\section{以“软度”衡量非晶态固体塑性的结构起源: 一种基于机器学习的定量理解}

刘晓俤 ${ }^{1}$, 李福成 ${ }^{1}$, 杨勇 $^{1,2 *}$

摘要 非晶态固体没有长程的平移对称性, 因而缺少像晶体中那样具有明确定义的缺陷及其运动来解释塑性的产生. 长期以来, 人们推测 非晶态固体的塑性可能起源于物理意义上的局部软区. 与此相比, 在Cubuk等人最近发表在《科学》杂志上的论文中, 作者基于机器学习 技术定义了一个微观结构量“软度”, 并提出可以通过“软度”来衡量多种不同非晶态固体(分子玻璃、胶体玻璃、金属玻璃等)的塑性的结 构起源. 尽管基于机器学习而得到的 “软度”的物理意义仍值得进一步研究, 但是由此得到的“软度”区域确实显示出与局部重排区域非常 好的相关性. 这个发现为探究多种非晶态固体塑性的结构起源提供了一个定量的理解. 